\title{
Endoneural Selective Stimulating Using Wire-Microelectrode Arrays
}

\author{
Jos P. A. Smit, Wim L. C. Rutten, and Herman B. K. Boom
}

\begin{abstract}
In acute experiments eight 5- to 24-wiremicroelectrode arrays were inserted into the common peroneal nerve of the rat, to investigate whether the electrodes could selectively stimulate motor units of the extensor digitorum longus (EDL) muscle. Twitch-force-recruitment curves were measured from the EDL for each array electrode. The curves were plotted on a double-logarithmic scale and parameterized by the low-force slope (which represents the power $p$ in the power-law relationship of force $F$ versus stimulus current $I$, or $F \sim I^{p}$ ) and the threshold current. The slopes and threshold currents measured with array electrodes did not differ significantly from those obtained with randomly inserted single wiremicroelectrodes. This indicates that, although involving a more invasive insertion procedure, electrode arrays provide neural contacts with low-force recruitment properties similar to those of single wires. Array results revealed partial blocking of neural conduction, similar to that reported with microneurographic insertion with single needles. The efficiency of the array was defined as the fraction of array electrodes selectively contacting a motor unit and evoking the corresponding threshold force. Efficiency thus expresses the practical value of the used electrode array in terms of the total number of distinct threshold forces that can be stimulated by selecting the appropriate electrodes. The eight arrays were capable of evoking threshold forces selectively with an average efficiency of 0.81 (or $81 \%$ ).
\end{abstract}

\section{INTRODUCTION}

A PPLICATION of artificial electrical stimulation for restoration of complex motor tasks, such as hand grasp or walking, requires selective control of the individual muscles involved in the task. Different levels of selectivity can be distinguished when electrically stimulating the peripheral neuromuscular system. Muscle selectivity implies control of a specific muscle without activating other muscles. This can be achieved with intramuscular electrodes or cuffs around the peripheral fascicle innervating only that muscle. Fascicle selectivity aims at stimulating a specific fascicle without activating other fascicles. To this end a cuff electrode can be used, placed around a peripheral fascicle, or around a nerve trunk containing multiple, clearly differentiated fascicles. Fascicle selectivity implies muscle selectivity when the activated fascicle innervates only one muscle. For fascicles innervating multiple muscles, fascicle selectivity provides a lower level of neuromuscular control than muscle selectivity. On the other hand, for fascicles innervating different parts

Manuscript received January 2, 1998; revised June 18, 1998, November 9, 1998, and March 8, 1999.

The authors are with the Faculty of Electrical Engineering, Institute for Biomedical Technology, University of Twente, Enschede 7500 AE The Netherlands.

Publisher Item Identifier S 1063-6528(99)09067-9. of one muscle, fascicle selectivity provides a higher level of neuromuscular control than muscle selectivity.

Size selectivity aims at activation of motor fibers with a specific diameter without activating other diameter fibers. It can not be compared to muscle selectivity or fascicle selectivity, since motor fibers with a specific diameter can innervate different muscles or occur in different fascicles. Size selectivity can not be achieved at will by distant electrodes or with a cuff electrode around the nerve. Only inverse recruitment is possible this way, i.e. with a distant electrode one may stimulate progressively thinner fibers with increasing current.

Fiber selectivity would enable the highest level of neuromuscular control. It implies that every motor fiber can be controlled independently of all other fibers. This might be achieved by placing electrodes inside the fascicle, i.e. closer to the nerve fibers than necessary for muscle or fascicle selectivity, but still extracellularly. We will refer to this as intrafascicular or endoneural selective stimulation, the subject of this paper.

Recent years have shown a growing interest in new ways of selective stimulation at the levels indicated above. Several methods have been reported which were classified as fascicle (or spatial) selective ([21], [23], [9]) or size selective ([1], [4], [5]). All of these stimulation methods have in common that they employ nerve cuff electrodes, and do not provide fiber selectivity. This implies that they can not be used to selectively activate an arbitrary nerve fiber (or spatially limited small group of fibers) within a fascicle. Therefore, these methods might not be suitable for generating finely graded movements while at the same time reducing fatigue.

It is obvious that endoneural selective stimulation of an arbitrary nerve fiber is best served by approaching that fiber very closely, preferably by putting an electrode in close proximity to one of its nodes of Ranvier [11], [17]. Experiments with endoneural microelectrodes support this idea, as they have shown that the recruitment behavior in the low-force range varies strongly with the position of a monopolar stimulating electrode [8], [13], [18], [19], [22]. Model simulations have confirmed this variation [8], [18]. The results of model studies presented by Meier et al. [12] and Frieswijk and Rutten [6] demonstrated that whether or not a nerve fiber is activated by a stimulus with a specific strength, strongly depends on the actual, specific geometrical arrangement of the nodes of Ranvier with respect to the electrode, thus causing differences in recruitment behavior (to be observed best in the low-force range) for different electrode positions. These findings at the 
same time suggest that selectivity of electrical stimulation can be increased by using as many endoneural electrodes as possible. Furthermore, both experimental and model results showed that an insulating extraneural medium generally leads to steeper recruitment curves and lower threshold currents than a well-conducting extraneural medium.

Literature on endoneural stimulation is scarce, and does not deal with selectivity at the fiber level. Nannini and Horch [14] tested the performance of intrafascicular recording electrodes as stimulating electrodes in peripheral nerves. Yoshida and Horch [25] studied selective stimulation of peripheral nerve fibers using dual intrafascicular electrodes. They were able to show fascicle selectivity as they independently activated separate fascicles, and to activate subpopulations within a single fascicle using pairs of electrodes in that fascicle. Recently, Yoshida and Horch [26] published a study on closed-loop control of ankle position using muscle afferent feedback with functional neuromuscular stimulation. They employed a dual channel intrafascicular electrode to stimulate a muscle and measured afferent information with two other dual channel intrafascicular electrodes. They were able to reach and maintain a fixed target ankle position in the presence of a varying external moment opposing the action of the ankle extensor, as well as track a sinusoidal target ankle position in the presence of a constant magnitude external moment.

As said, the ultimate case of selectivity would result if it were possible to stimulate each motor fiber independently. In this ideal situation the minimum number of electrodes would equal the number of motor fibers to be activated. Since motor fiber activation is established at a node of Ranvier [11], [17], these electrodes should be arranged in a specific threedimensional pattern, such that the first node of Ranvier activated by each electrode belongs to a different motor fiber for each electrode. As the actual positions of the nodes of Ranvier are not known, this prerequisite will be hard to meet. Therefore, selective stimulation of arbitrary motor fibers (or even a spatially limited small group of them) requires an endoneural multi-electrode with a redundant number of electrodes.

Such multi-electrodes for stimulation are under development but not yet available [7], [18] (see also reports on similar devices for recording, for example [10]). However, analogously dimensioned one- and two-dimensional wire-microelectrode arrays (WMEA's) were constructed and, via an incision in the nerve sheath, inserted into rat peroneal nerve. The objective of the experiments was to analyze force-recruitment curves and thresholds, in order to monitor selectivity and efficiency of endoneural stimulation.

It is important to consider the influence of an epineural/perineural incision. Two possible effects may be discerned. First of all, creation of an incision will affect neuromuscular metabolism (due to damage to epineurium, perineurium and, possibly, blood supply), and thus viability of motor units. Furthermore, an incision will introduce a local "fluid leak" in the less-well conducting sheaths surrounding fascicle and nerve. This can give rise to current shunting via fluid accumulating in the incision, thus raising stimulation thresholds.

Besides presentation and analysis of recruitment data from the multi-electrode arrays, short-term (several hours) stability of the electrode-nerve contact is investigated and topographical recruitment-curve maps are presented. The low-force range of the curves is characterized by their slope and threshold current. To evaluate possible insertion side-effects, slopes and thresholds are statistically compared to results from (less invasive) single wire-microelectrodes (SWME's). Also, on a prolonged time scale (up to four hours), the time course of maximum muscle force is examined.

Also, most importantly for future applications, attention is paid to efficiency (i.e., the percentage of electrodes in the array contacting a distinct motor unit) and selectivity (i.e., the percentage of motor units that can be distinctly contacted by using different electrodes in the array). The low-force recruitment range is again of prime importance in this context, as this is the practical operating range for individual electrodes in an endoneural stimulation device.

\section{METHODS}

\section{A. Experimental Procedure}

Acute experiments were conducted on male Wistar rats (3-8 months old, 300-500 g weight) maintained under sodium pentobarbital anesthesia (Nembutal, initial dose: $70 \mathrm{mg} / \mathrm{kg}$ I.P., maintenance level: $20-35 \%$ of initial dose repeated every hour). The animal was placed on a heated table kept at $38 \pm$ $0.5^{\circ} \mathrm{C}$. Both tendons of the extensor digitorum longus (EDL) muscle of the right hind leg were cut. To ensure isometric conditions the proximal tendon was mechanically fixed and the distal tendon connected to an isometric force transducer. The muscle (not the nerve) was regularly covered with a thin layer of paraffin oil to prevent it from drying out. The peroneal branch (which innervates the EDL muscle) of the exposed sciatic nerve was prepared free. The tibial branch was crushed with a suture. All exposed body tissues were maintained at $38 \pm 1{ }^{\circ} \mathrm{C}$ by a warm air stream almost saturated with water vapor [24]. Care was taken to avoid water condensation on the nerve.

After positioning the animal, an $\mathrm{Ag} / \mathrm{AgCl}$ reference electrode was inserted into the gastrocnemius muscle. A hook electrode was placed around the common peroneal nerve, and supramaximal stimuli were applied in order to determine the optimal twitch length (OTL, i.e., the length of the muscle where twitch force is maximal) and the initial maximum twitch-contraction force of the EDL muscle. The hook electrode was then removed.

The common peroneal nerve was now placed on a support table and an incision was made using a pair of tweezers and an ophthalmic knife. The incision was directed along the longitudinal axis of the nerve and long enough to allow easy insertion of a WMEA. Protrusion of the endoneurium due to intrafascicular pressure was observed in approximately $75 \%$ of the incisions made. A one-dimensional five-channel array and a two-dimensional 24-channel array were used, consisting of five rows of one electrode and six rows of four electrodes, respectively. Fig. 1(a) shows a scanning electron micrograph of the 24-channel array. The individual electrodes in the arrays were $25 \mu \mathrm{m}$-diameter $\mathrm{NiCr}$ wires insulated with a $4 \mu \mathrm{m}$ Karma 


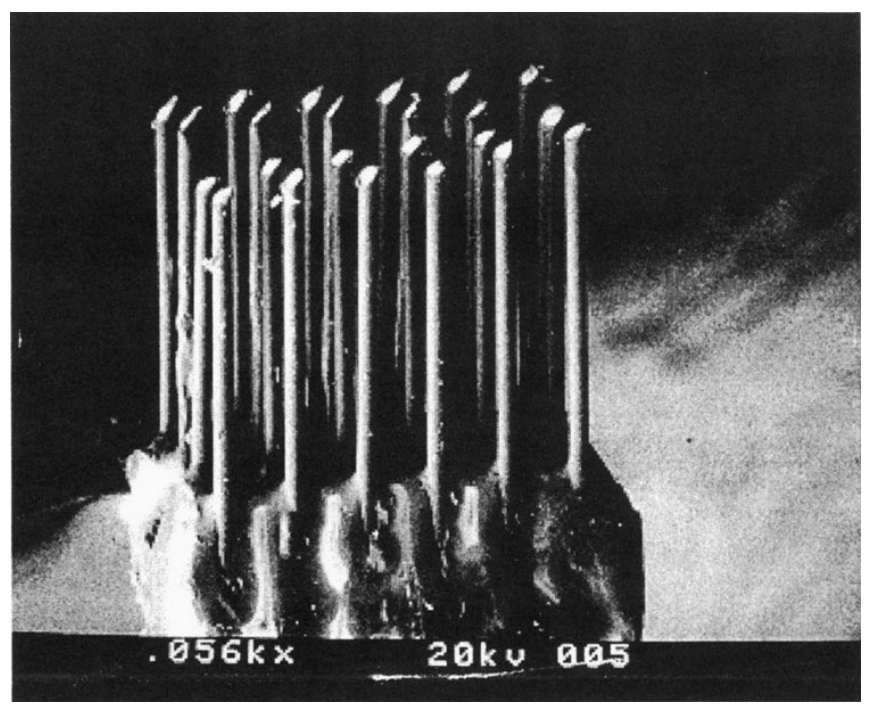

(a)

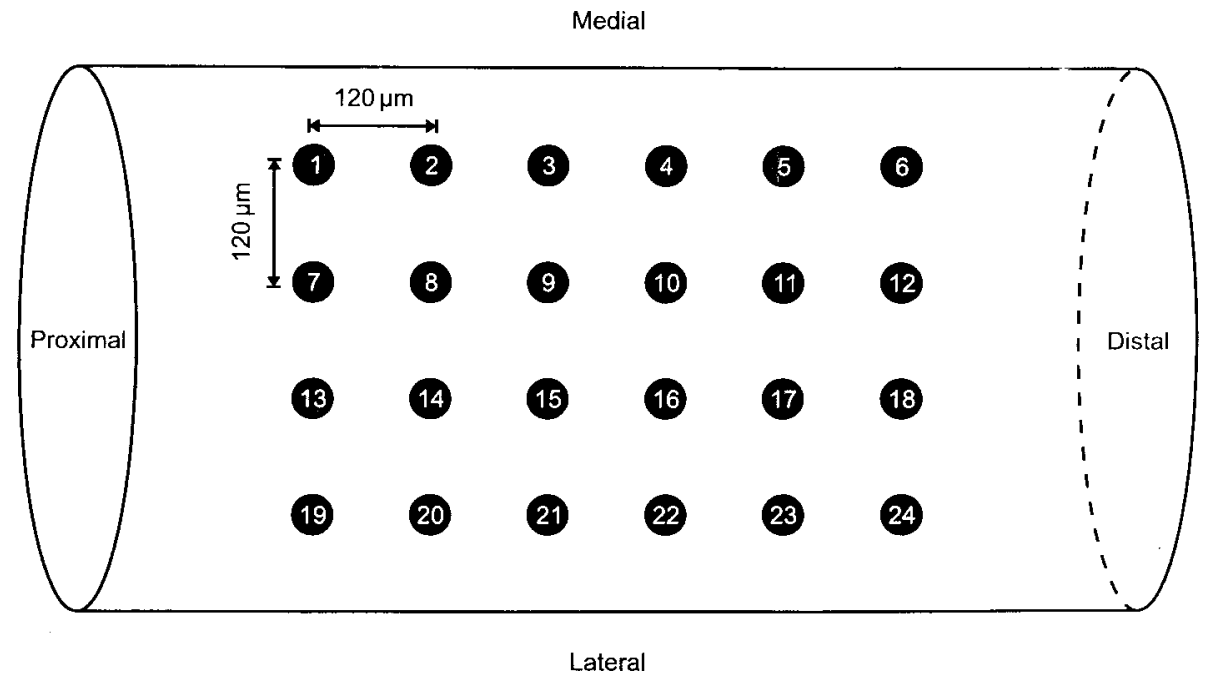

(b)

Fig. 1. (a) Scanning electron micrograph of the 24-channel wire-microelectrode array. The individual electrodes are located at the exposed tips of insulated $\mathrm{NiCr}$ wires with a diameter of $25 \mu \mathrm{m}$. Interelectrode spacing is $120 \mu \mathrm{m}$. (b) Diagram of the electrode positions (black numbered circles) relative to the common peroneal nerve.

coating (California Fine Wire Co., Grover City, CA). Only the obliquely cut tip was uninsulated, resulting in an effective electrode area of about $2800 \mu \mathrm{m}^{2}$ and electrode impedance of about $1 \mathrm{M} \Omega(f=1 \mathrm{kHz}, I<1 \mu \mathrm{A})$. Interelectrode spacing was $120 \mu \mathrm{m}$. Fig. 1(b) shows diagram of the electrode positions relative to the common peroneal nerve.

After insertion into the nerve, the WMEA was allowed to settle for approximately 30 minutes. Then, rectangular depolarizing current pulses of $100 \mu$ s duration were generated by a home-built, computer-controlled stimulator. For each electrode in the array a series of stimuli was applied with amplitudes increasing from subthreshold to supramaximal. Stimulus current step size was $0.1 \mu \mathrm{A}$ or $0.2 \mu \mathrm{A}$; maximum stimulus current varied from $30 \mu \mathrm{A}$ to $100 \mu \mathrm{A}$. Stimulus repetition rate was $1 \mathrm{~Hz}$ to preclude fatigue and potentiation effects [2]. The elicited twitch-contraction forces were measured, one for each stimulus amplitude. Recruitment curves were constructed off-line by determining the peak values of the collected twitch force data.

Twitch forces were measured using a Model 373 Isometric Force Transducer (Harvard Apparatus Company, Inc., Millis, MA). The sensitivity of this transducer is $2 \mathrm{mV} / \mathrm{mN}$ over a range from 4.9 to $1078 \mathrm{mN}$; accuracy is $1.25 \mathrm{mN}$. The force signal was amplified two times and filtered $(0-500 \mathrm{~Hz}, 50 \mathrm{~Hz}$ notch), and then sampled at $2 \mathrm{kHz}$. The overall resolution of the force measurement system was $0.31 \mathrm{mN} / \mathrm{b}$.

A recruitment curve was measured for each of the electrodes in the array. The electrodes were operated sequentially in increasing electrode number, from electrode 1 to electrode 24 [cf., Fig. 1(b)]. The muscle was allowed two minutes of rest between recruitment curves for consecutive electrodes. The nerve was kept moist by applying Ringer's solution at regular intervals. Around the incision only small quantities of Ringer were used to minimize the risk of introducing a current shunt 
between electrodes and extraneural medium. The OTL was checked regularly during the experiment.

At the end of the experiment, the electrode array was carefully removed from the nerve and the quality of insertion visually assessed. We generally found that the electrode array was firmly attached to the nerve and the electrodes were clearly positioned inside the fascicle. In approximately $17 \%$ of the cases, however, the array had not been inserted properly and the electrodes had not entered the fascicle. These experiments were excluded from further analysis.

Finally, the hook electrode was placed around the nerve again, and the post-experiment maximum twitch-contraction force determined by applying supramaximal stimuli. Electrode positions proximal and distal to the incision site were evaluated. The hook electrode was then removed.

\section{B. Data Analysis}

In order to compare the recruitment behavior in the lowforce range, the recruitment curves were plotted on a doublelogarithmic scale and parameterized by the threshold current and the slope of the first-order fit to the recruitment data in the low-force range. Analogously to the analysis in [8], the low-force range was defined as the range between the noise threshold of $4.9 \mathrm{mN}$ and $147 \mathrm{mN}$. The latter limit was determined empirically to provide exclusion of the highforce saturation region in the recruitment curves from the fitting procedure. Threshold current was defined as the lowest stimulation current that resulted in a twitch-force amplitude above the noise threshold. Slope was determined by fitting the double-logarithmic data to a straight line by linear regression. Therefore, the slope represents the power $p$ in the power-law relationship $F \sim I^{p}$, where $F$ is twitch-force maximum and $I$ is stimulus current amplitude.

A measure of efficiency $E$ can be defined as the ratio of the number of electrodes selectively addressing a threshold force $\left(N_{E L t h r}\right)$ and the total number of electrodes in the array $\left(N_{\text {ELtot }}\right)$

$$
E=N_{E L t h r} / N_{E L t o t}
$$

Efficiency thus expresses the practical value of the used electrode array in terms of the total number of distinct threshold forces that can be stimulated by selecting the appropriate electrodes.

Note that, since the force transducer employed in the experiments only allowed detection of forces in the range from 4.9 to $1078 \mathrm{mN}$ with an accuracy of $1.25 \mathrm{mN}$, it was not possible to identify the smallest motor units activated in the experiments. Therefore, the actual number of motor units contacted selectively with an electrode array could not be determined. It was possible, however, to determine the number of threshold forces above $4.9 \mathrm{mN}$. These threshold forces may thus be either single motor unit forces or the combined contributions of several units.

A measure of selectivity $S$ can be defined as the ratio of the number of distinct motor units that can be activated by the electrode array $\left(N_{M U d i s}\right)$ and the total number of motor units to be controlled $\left(N_{M U t o t}\right)$

$$
S=N_{\text {MUdis }} / N_{\text {MUtot }}
$$

Selectivity thus expresses the potential of the specific configuration of the used stimulation device (in terms of number and spacing of electrodes) for addressing distinct motor units.

A measure of selectivity would also be obtained by applying the method as used earlier [17], in which stimulus field overlaps of two electrodes are evaluated from the addition of their respective forces, using the refractive property of nerve fibers. However, this method is too time-consuming for the present multi-electrodes.

\section{RESULTS}

\section{A. Short-Term Stability of the Electrode-Nerve Contact}

In three animals we assessed the short-term stability of the electrode-nerve contact. Fig. 2 shows one example of a timeseries of log-log recruitment curves elicited by one of the 24 electrodes. The curves are recorded sequentially in time, at $t=0,10,20,30,45,60,75,90,105,120,135,150,165$, and $180 \mathrm{~min}$. The applied stimulus current range was identical for all curves, 1-10 $\mu \mathrm{A}$, and is indicated by the horizontal scale bar above each curve. For clarity, the curves are shifted arbitrarily along the horizontal axis, but proportional to their time of measurement.

The forces in the $6 \mathrm{mN}$-range represent the first levels exceeding the force transducer's noise threshold. When stimulus strength is increased beyond the threshold level, an additional (group of) motor unit(s) is recruited. This results in a relatively large force increment, accumulating in a force plateau at 17 $\mathrm{mN}$. The shape of the recruitment curve in the low-force range is stable up to approximately 135 minutes and, most importantly, the force plateau at $17 \mathrm{mN}$ is stable, indicating that the position of the stimulation electrode was stable.

For longer time periods a horizontal shift to lower current values in the low-force range becomes prominent. Forces below $10 \mathrm{mN}$ shift "out of sight" (i.e. to current values below 1 $\mu \mathrm{A})$, as does eventually the $17 \mathrm{mN}$ plateau. At the same time, the shift below is counterbalanced by longer "tails" above, at maximum force. This effect, being the opposite of current shunting through presence of excess fluid in the incision area, indicates that with time current shunt leaks diminish at the site of incision. This same process eventually will result in the electrode array becoming more firmly attached to the nerve.

\section{B. Topographical Recruitment-Curve Maps}

A total of 102 recruitment curves was measured from six animals. The five-channel electrode array was used in one animal, at three different positions along the nerve. The 24-channel electrode array was used in five animals; only one array position was evaluated for these animals. Consequently, eight sets of recruitment curves were obtained in total (Table I). In Fig. 3, a complete set of 24 recruitment curves is shown, measured at one array position in one animal. Note the different curve shapes in the low-force range. These reflect different recruitment behavior for different electrode positions. 


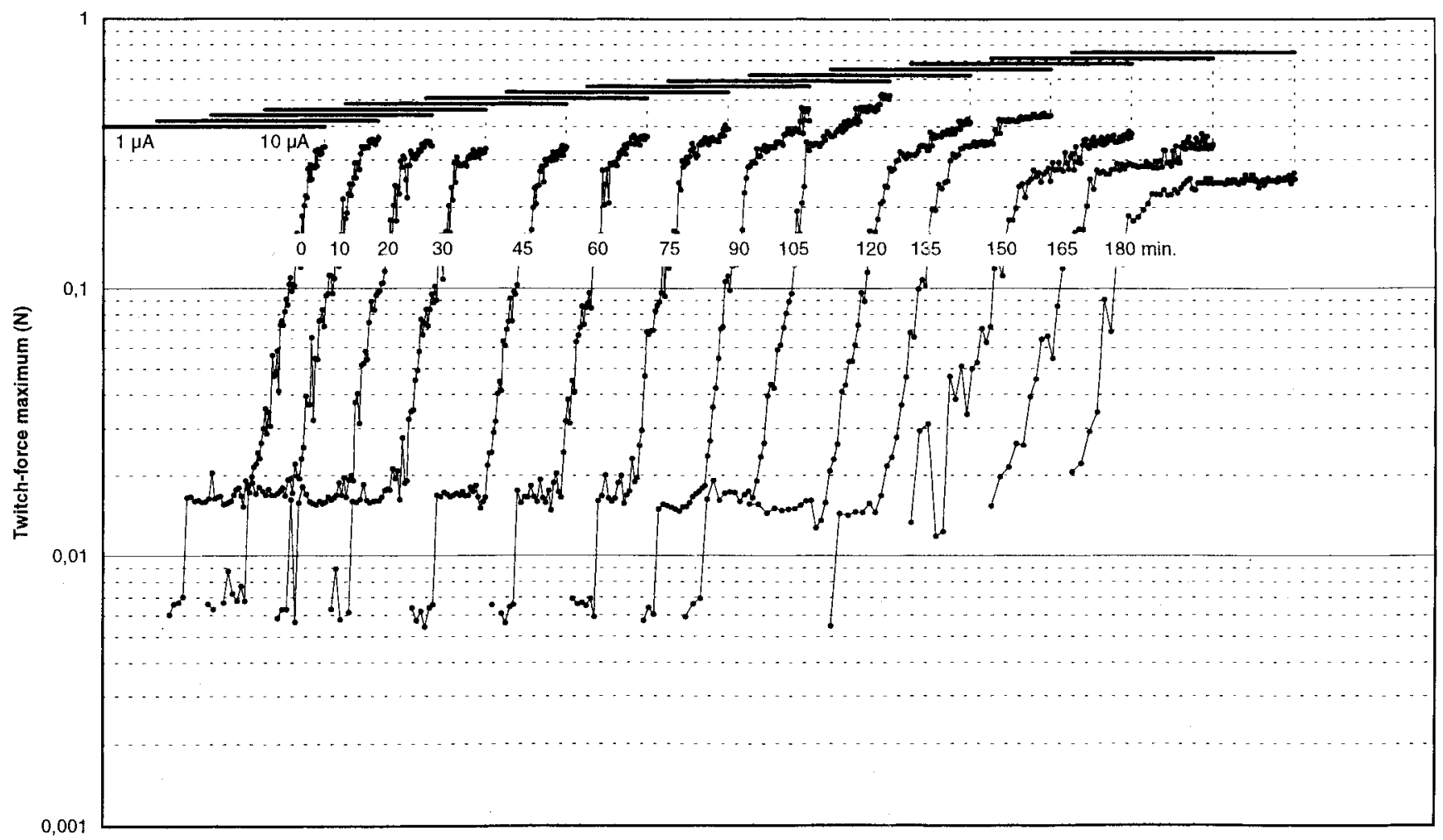

Fig. 2. Log-log recruitment curves measured for a single electrode in the 24-channel wire-microelectrode array at different points in time in one animal. Left to right: $t=0,10,20,30,45,60,75,90,105,120,135,150,165$, and $180 \mathrm{~min}$. Stimulus current range was $1-10 \mu \mathrm{A}$ for all curves, as indicated by the horizontal scale bars associated with each curve. For clarity, the curves are shifted arbitrarily along the horizontal axis, but proportional to their time of measurement.

TABLE I

Compilation of Relevant Data Pertaining to the WMEA Experiments. A Total of 102 Recruitment Curves was Measured from Six Animals. These Curves Are Arranged in Eight Sets: Three Complete Sets of 5 Curves, Two Complete Sets of 24 Curves, and Three Partial Sets Due to the Premature Death of the Animal. Experiments A, B, and C Were Conducted on the Same Animal, i.e., with the Electrode Array Inserted at Three Different Positions Along the Nerve

\begin{tabular}{ccccc}
\hline Rat no. & Experiment & Array type & No. of curves & Electrodes used \\
\hline 1 & a & $5 \times 1$ & 5 & $1-5$ \\
1 & b & $5 \times 1$ & 5 & $1-5$ \\
1 & c & $5 \times 1$ & 5 & $1-5$ \\
2 & d & $6 \times 4$ & 24 & $1-24$ \\
3 & c & $6 \times 4$ & 8 & $1-8$ \\
4 & f & $6 \times 4$ & 17 & $1-14$ \\
5 & g & $6 \times 4$ & 24 & 14 \\
\hline 6 & h & $6 \times 4$ & & 14 \\
\hline
\end{tabular}



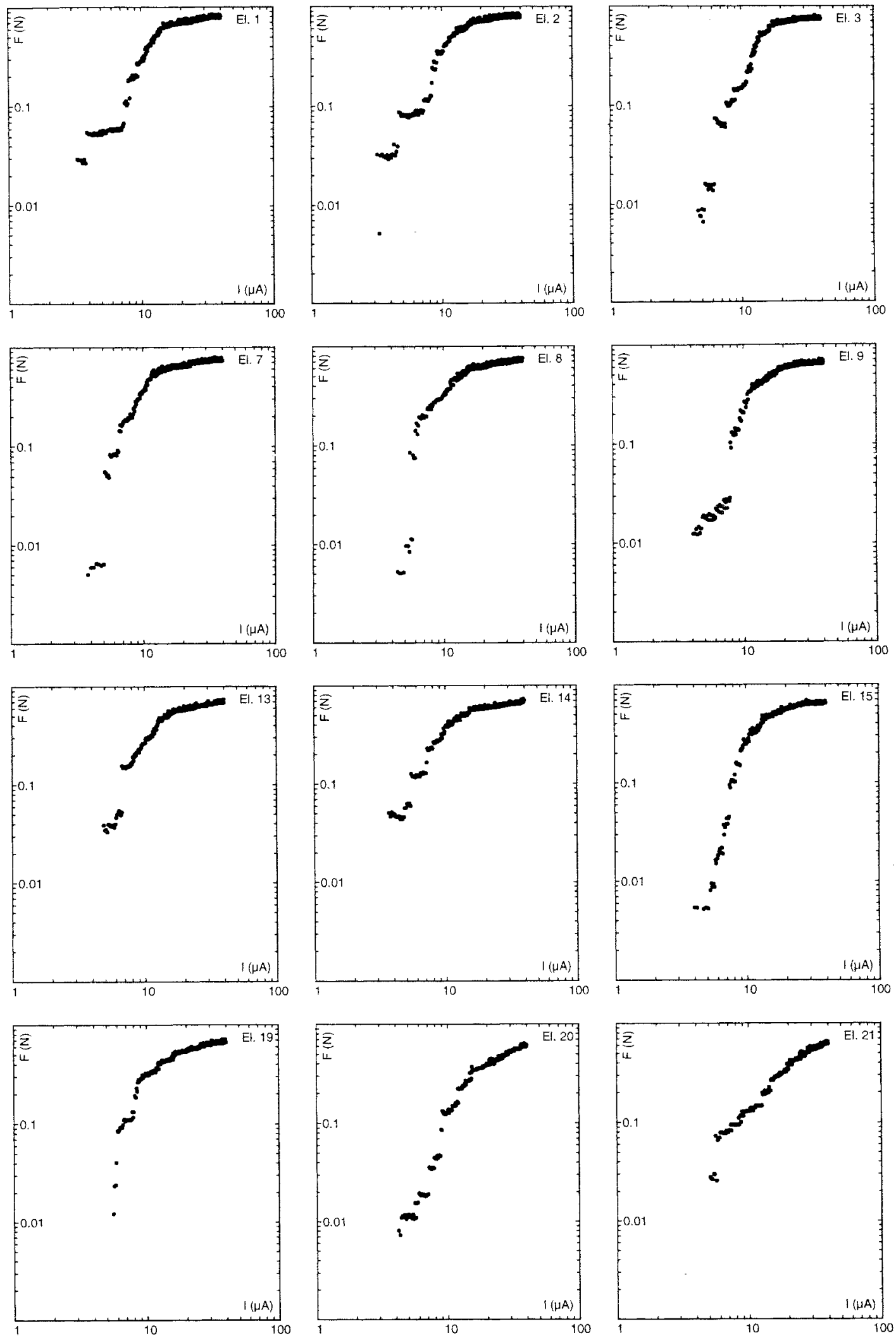

(a)

Fig. 3. Topographical map of recruitment curves obtained from one animal for a fixed position of the 24-channel wire-microelectrode array (experiment g). Each curve corresponds to a single electrode and is the result of applying a series of single stimuli with amplitudes increasing from 1 to $40 \mu \mathrm{A}$ in steps of $0.1 \mu \mathrm{A}$. The electrodes were operated sequentially in increasing electrode number, from electrode 1 to electrode 24 [cf., Fig. 1(b)]. The noise threshold of the force transducer employed lies at $4.9 \mathrm{mN}$. Data below this level have not been shown. The geometrical arrangement of the curves corresponds to the positions of the electrodes in the array $(6 \times 4$ electrodes, cf., Fig. 1). Electrode numbers are indicated in the upper right corner for each curve. Top to bottom: recruitment curves for electrodes positioned along the radial direction of the nerve (top: medial; bottom: lateral). Left to right: recruitment curves for electrodes positioned along the longitudinal direction of the nerve (left: proximal; right: distal). 

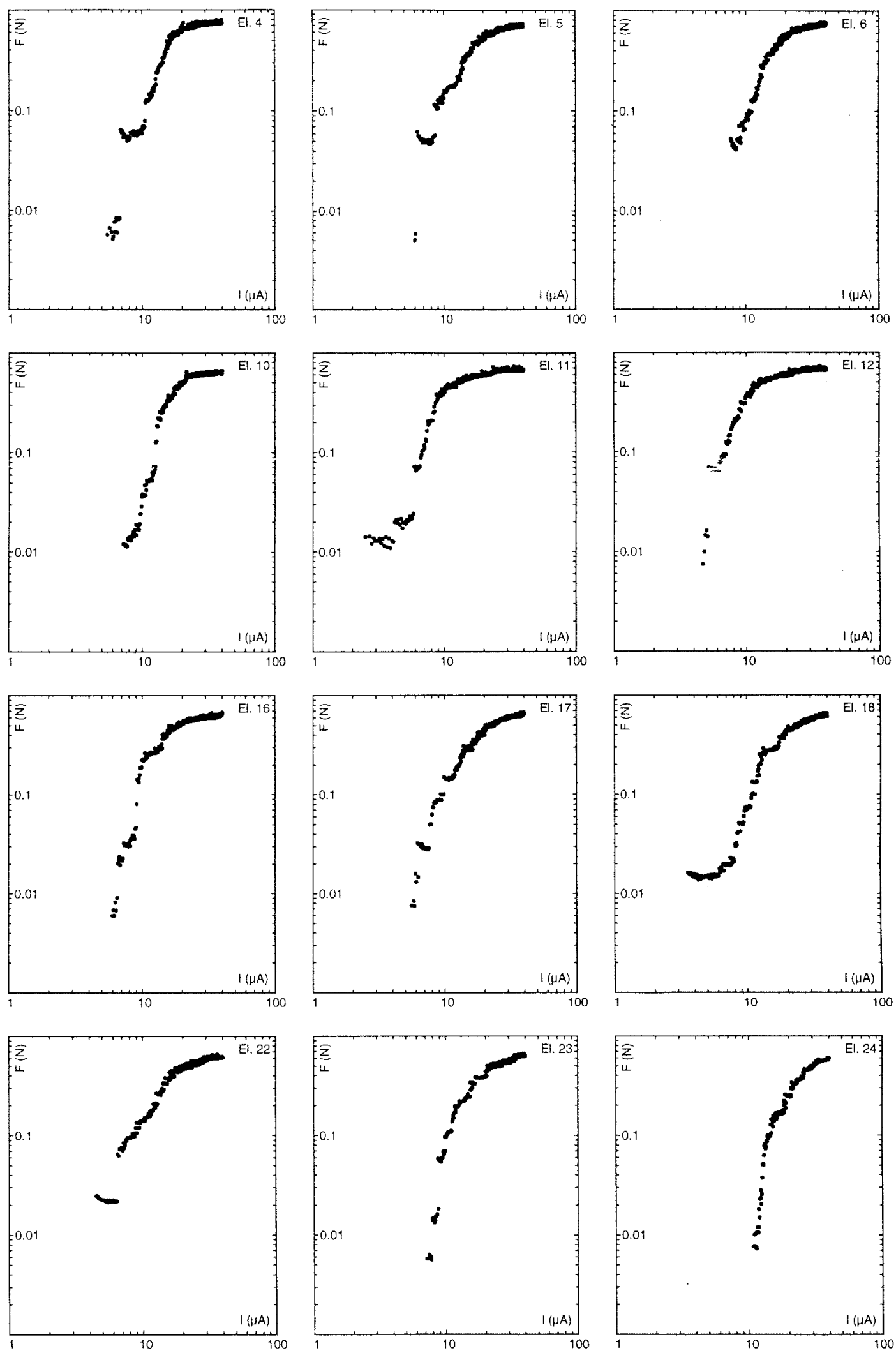

(b)

Fig. 3. (Continued.) Topographical map of recruitment curves obtained from one animal for a fixed position of the 24-channel wire-microelectrode array (experiment g). Each curve corresponds to a single electrode and is the result of applying a series of single stimuli with amplitudes increasing from 1 to 40 $\mu \mathrm{A}$ in steps of $0.1 \mu \mathrm{A}$. The electrodes were operated sequentially in increasing electrode number, from electrode 1 to electrode 24 [cf., Fig. 1(b)]. The noise threshold of the force transducer employed lies at $4.9 \mathrm{mN}$. Data below this level have not been shown. The geometrical arrangement of the curves corresponds to the positions of the electrodes in the array $(6 \times 4$ electrodes, cf., Fig. 1). Electrode numbers are indicated in the upper right corner for each curve. Top to bottom: recruitment curves for electrodes positioned along the radial direction of the nerve (top: medial; bottom: lateral). Left to right: recruitment curves for electrodes positioned along the longitudinal direction of the nerve (left: proximal; right: distal). 


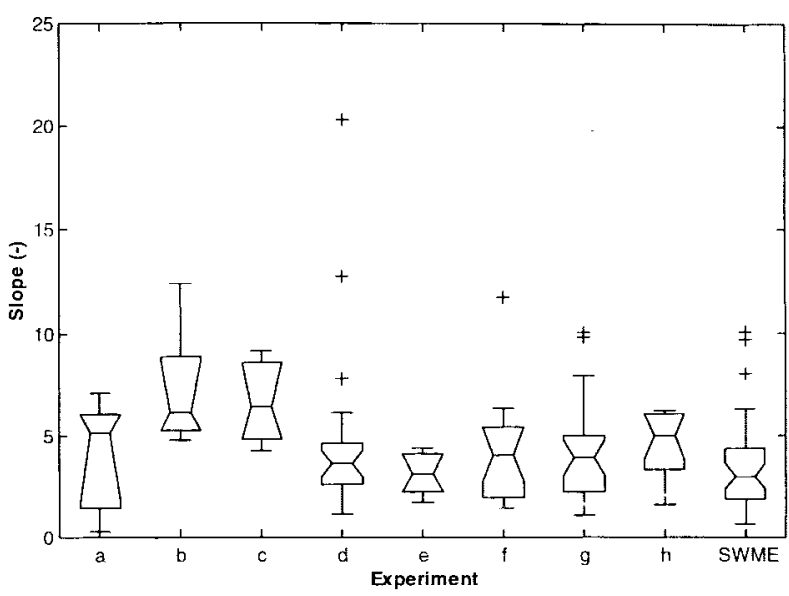

(a)

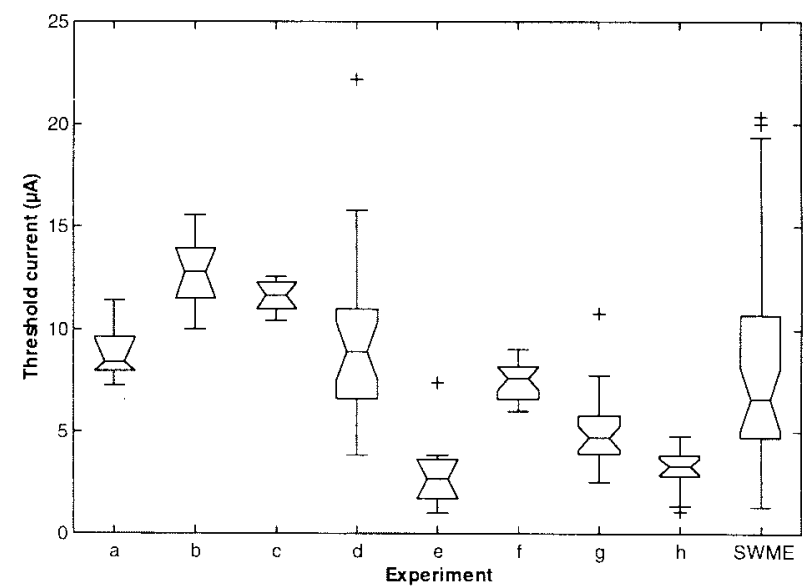

(b)

Fig. 4. Box plots showing low-force slopes (which represent the power $p$ in the power-law relationship $F \sim I^{p}$ ) (a) and threshold currents (b) of the force-recruitment curves for the WMEA experiments listed in Table I and for the SWME experiments presented in [8] for a nerve immersed in Ringer's solution. The lower and upper lines of the "boxes" indicate the 25th and 75th percentile for each of the data sets. The distance between the top and the bottom of the boxes is the interquartile range. The lines in the middle of the boxes indicate the mean for each of the data sets. The "notches" in the boxes indicate the $95 \%$-confidence interval for the mean. Outliers (marked by "+"-symbols) were defined as values that are more than 1.5 times the interquartile range away from the top or the bottom of the box. The extents of the rest of the sample are indicated by the "whiskers."

\section{Slopes and Threshold Currents}

Fig. 4 gives a graphical representation of low-force slopes and threshold currents for the eight sets of WMEA data. Correlation coefficients for the first-order fits ranged from 0.67 to 0.98 (median: 0.92). The data for the single wiremicroelectrode experiments presented in [8] for a nerve immersed in Ringer's solution, have been added for comparison $(n=39)$.

Set $b$ and set $c$ show large mean slopes (7.3 and 6.7, respectively). The remaining sets have mean slopes smaller than five. Mean threshold currents are smaller than $10 \mu \mathrm{A}$, except for set $b$ and set $c$.

Set $b$ and set $c$ contain only five recruitment curves, measured for five electrodes positioned along the nerve. The high mean threshold currents might be an indication that the electrodes were relatively far away from the nodes of Ranvier (even though the quality of insertion was considered good upon removal of the electrode array). This is consistent with the high mean slopes for set $\mathrm{b}$ and set $\mathrm{c}$.

The eight sets of WMEA data were compared mutually in order to reveal inter-set differences. To this end, a one-factor ANOVA was employed to test the null hypothesis $\left(H_{0}\right)$ that there is no difference between the eight means, both for the slopes and the threshold currents.

At the 5\%-level of significance the means of the slopes reveal no differences $(p=0.182)$, but the means of the threshold currents do $(p \approx 0)$. As each set is characterized by a combination of mean slope and mean threshold current, these results indicate that the eight sets are different. This discrepancy must probably be ascribed to the fact that the threshold measure relies on a rather small number of data (see Discussion).

In order to obtain a set of WMEA data of sufficient sample size, the data from the eight WMEA experiments were pooled in a similar fashion as the SWME results in [8]. This enables a comparison of the WMEA results $(n=102)$ with the SWME results $(n=39)$, both groups containing data from multiple electrode positions in multiple animals.

A two-sample, two-tailed $F$-test was performed on the variances of both slopes and threshold currents of WMEA's and SWME's. For a 1\%-level of significance we found no differences in the variances of the slopes $(p=0.050)$. The variances of the threshold currents are significantly different, however $(p=0.002)$.

Two two-sample, two-tailed $t$-tests (assuming equal variances for slopes and unequal variances for threshold currents) showed no differences in the means of slopes $(p=0.028)$ and threshold currents $(p=0.121)$ for the WMEA's and the SWME's (1\%-level of significance).

\section{Time Course of Maximum Muscle Force}

The WMEA experiments are rather lengthy. Due to the prolonged exposure of nerve and muscle to nonideal physiological conditions, (some) tissue deterioration may occur. This might have consequences for the sets of recruitment curves obtained during the experiments. As a monitor for this, we evaluated the time course of the maximum muscle force.

The time interval between recording two consecutive recruitment curves was approximately ten minutes. For the first and the last curve measured for the 24-channel electrode array this thus accumulated to $230 \mathrm{~min}$. Fig. 5 shows the time course of maximum (twitch-)force and corresponding stimulation current for the set of recruitment curves in Fig. 3.

The maximum force has a clear negative trend with electrode number (and thus with time), whereas the current at which maximum force is attained is nearly constant among electrodes [Fig. 5(a)]. Also, the threshold current does not show a clear trend [Fig. 5(b)]. This was generally observed among the eight sets of recruitment curves. The decrease of maximum force is thus caused by deterioration of muscle and/or nerve, rather than by current shunting through the incision. Note that a similar decrease of maximum force with time is also visible in Fig. 2. 


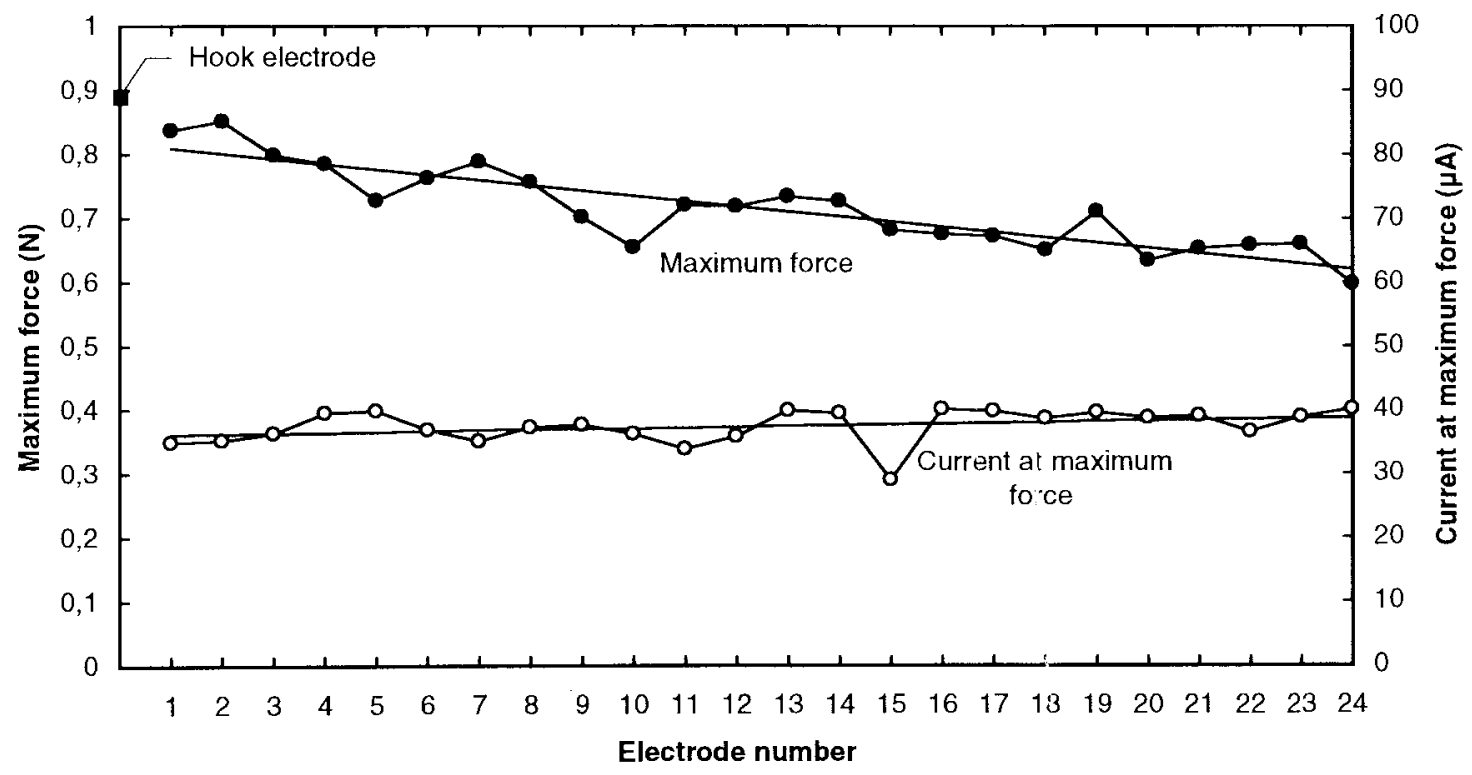

(a)

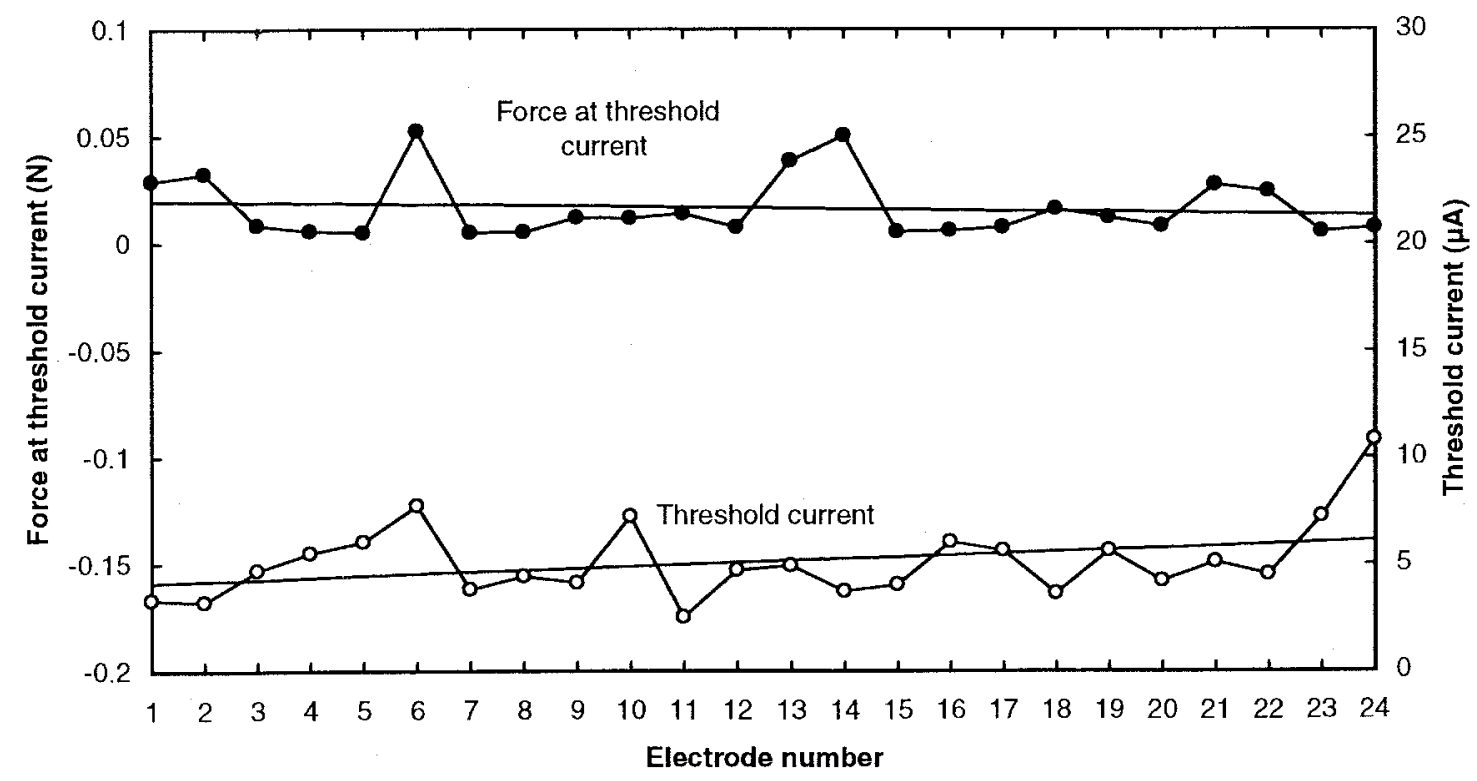

(b)

Fig. 5. Time course of twitch-force maximum and corresponding stimulation current for the recruitment curves obtained in experiment g. (a) Maximum force (left axis) and current at maximum force (right axis) as a function of electrode number. (b) Threshold current (right axis) and force at threshold current (left axis) as a function of electrode number. The time interval between the data for two consecutive electrodes is approximately ten minutes. The first-order fits to the data are also shown. The black square on the left axis in (a) indicates the initial maximum force as obtained from stimulation with a hook electrode prior to incising the nerve.

The maximum forces for the electrode array are all lower than the initial maximum force as obtained from stimulation with a hook electrode prior to incising the nerve [Fig. 5(a)]. This suggests that creation of an incision results in some loss of force. However, the recruitment curve for the first electrode was measured approximately $60 \mathrm{~min}$ subsequent to that for the hook electrode. Therefore, muscle and/or nerve deterioration might also have played a role in this initial force loss.

At the end of the experiments, after removal of the electrode array, the maximum force for stimulation with a hook electrode proximal to the incision was generally lower than for stimulation distal to the incision. Apparently, at the site of the incision propagation of action potentials toward the muscle is partially blocked. Both maximum forces were lower than the initial maximum force attained prior to incising the nerve. This again must be ascribed to deterioration of muscle and/or nerve.

Threshold current and force at threshold current vary among electrodes, but do not reveal a clear trend [Fig. 5(b)]. This variation is due to the fact that these parameters are mainly determined by the position of the first node of Ranvier activated for each electrode, and the strength of the corresponding motor unit. 
TABLE II

Threshold Forces and Associated Threshold Currents for Experiment $g$ (cf., Table I). The Electrodes Are Ordered According to Increasing Threshold Forces. The Horizontal Lines Indicate a Classification of the Data into $2.5 \mathrm{mN}$-Wide Bins as Imposed By Force-Transducer Accuracy. The Bin Limits Are Given By $2.5 \cdot i \pm 1.25 \mathrm{mN}(i=0,1,2, \ldots)$

\begin{tabular}{|c|c|c|c|}
\hline Bin limits $(\mathrm{mN})$ & Electrode no. & Threshold current $(\boldsymbol{\mu} \mathbf{A})$ & Threshold force $(\mathrm{mN})$ \\
\hline \multirow[t]{7}{*}{$5.0 \pm 1.25$} & 5 & 6.0 & 5.02 \\
\hline & 7 & 3.8 & 5.02 \\
\hline & 8 & 4.4 & 5.20 \\
\hline & 15 & 4.0 & 5.38 \\
\hline & 4 & 3.5 & 5.62 \\
\hline & 23 & 7.2 & 5.74 \\
\hline & 16 & 6.0 & 5.98 \\
\hline \multirow[t]{5}{*}{$7.5 \pm 1.25$} & 12 & 4.7 & 7.42 \\
\hline & 17 & 5.6 & 7.54 \\
\hline & 24 & 10.8 & 7.66 \\
\hline & 20 & 4.2 & 8.02 \\
\hline & 3 & 4.7 & 8.49 \\
\hline \multirow[t]{3}{*}{$12.5 \pm 1.25$} & 10 & 7.2 & 11.96 \\
\hline & 19 & 5.6 & 12.14 \\
\hline & 9 & 4.1 & 12.26 \\
\hline \multirow[t]{2}{*}{$15.0 \pm 1.25$} & 11 & 2.5 & 14.00 \\
\hline & 18 & 3.6 & 16.03 \\
\hline $25.0 \pm 1.25$ & 22 & 4.5 & 24.58 \\
\hline $27.5 \pm 1.25$ & 21 & 5.1 & 27.57 \\
\hline $30.0 \pm 1.25$ & 1 & 3.3 & 29.13 \\
\hline $32.5 \pm 1.25$ & 2 & 3.2 & 32.36 \\
\hline $37.5 \pm 1.25$ & 13 & 4.9 & 38.70 \\
\hline $50.0 \pm 1.25$ & 14 & 3.7 & 50.36 \\
\hline $52.5 \pm 1.25$ & 6 & 7.7 & 52.40 \\
\hline
\end{tabular}

\section{E. Efficiency of Stimulation}

The efficiency of endoneural stimulation has been defined as the number of distinct threshold forces that can be contacted per electrode in the array.

Analysis of the 24 recruitment curves in Fig. 3 resulted in the 24 threshold forces shown in Table II. The electrodes are listed in order of increasing threshold force. Because of limited force-transducer accuracy (see Section II), the electrodes should be grouped into $2.5 \mathrm{mN}$-wide bins. These are indicated by horizontal lines. All threshold forces contained in one such bin must be considered identical, since force-transducer accuracy does not allow discrimination between them.
Table II reveals 11 different bins. This implies that the 24 recruitment curves in Fig. 3 contain 11 different threshold forces. So, for 13 of the 24 electrodes, the observed threshold force was similar to that seen at one or more of the other electrodes. Therefore, the efficiency equals

$$
E=11 / 24=0.46 \text {. }
$$

\section{DISCUSSION}

\section{A. Slopes and Threshold Currents}

The recruitment curves were parameterized by the slope in the low-force range and the threshold current. This was 
done to obtain an objective, yet simple, characterization of the low-force recruitment behavior in endoneural stimulation. It is difficult to compare the values encountered in the present study with data from other investigators, as literature on force recruitment generally provides too few data points for proper estimation of these parameters.

The statistical analysis reveals no significant difference between recruitment curves measured with WMEA's and SWME's, as far as mean slopes and mean threshold currents are concerned. This means that WMEA's provide neural contacts with low-force recruitment properties similar to those of SWME's. This might be an indication that the epineu$\mathrm{ral} /$ perineural incision, necessary for array insertion, does not adversely affect the recruitment behavior (see also further).

Mean slopes are generally smaller than five, and mean threshold currents smaller than $10 \mu \mathrm{A}$, except for experiments $\mathrm{b}$ and $\mathrm{c}$ (Fig. 4). This combination of slopes and threshold currents is an indication that for the latter two sets the electrodes were relatively far away from the nodes of Ranvier (even though the quality of insertion was considered good upon removal of the electrode array).

For the standard deviations of the slopes the most striking feature is the relatively large value for set d. From the box plot in Fig. 4(a) it can be seen that this is mainly caused by two outliers (corresponding to electrodes 21 and 22) with values larger than ten. The large values might indicate that the electrodes were at a relatively large distance from the nearest nodes of Ranvier, consistent with the fact that the threshold currents for electrode $21(15.8 \mu \mathrm{A})$ and electrode $22(22.2 \mu \mathrm{A})$ are considerably larger than those for the rest of the electrodes in the array (generally less than $10 \mu \mathrm{A}$ ).

Fig. 4(b) also reveals a large standard deviation for the threshold current of the SWME data. This set contains data from 39 recruitment curves measured for randomly inserted SWME's in ten animals [8]. The electrode tip positions of the 24-channel WMEA sample the nerve space less randomly (i.e. they span a plane of about $600 \times 360 \mu \mathrm{m}$ ). Probably, this results in less variation of thresholds currents for the WMEA sets.

\section{B. Time Course of Maximum Muscle Force}

The negative trend of maximum force with electrode number (as observed for the eight sets of recruitment curves) was attributed to deterioration of muscle and/or nerve, rather than to current shunting through the incision. This can be understood by considering that body tissues were exposed to nonideal physiological conditions for six to eight hours during the experiments.

Fatigue must be considered a factor of minor influence in this process, since stimulus repetition rate was kept as low as 1 Hz. Furthermore, the muscle was allowed to recover for 2 min between measurement of two consecutive recruitment curves.

Loss of maximum force might also have been the result of the endoneural electrodes not activating all fibers, in contrast to the hook electrode. This factor must be excluded, however, as supramaximal stimuli (up to $200 \mu \mathrm{A}$ ) were applied regularly during the experiments to ensure that maximum force was actually reached in each recruitment curve.

\section{Blocking of Action Potentials}

Although not very relevant for stimulation of peripheral nerve in case of spinal cord lesion, introduction of electrodes into nerves may cause action potential blocking [3], [16]. We looked briefly into this aspect.

At the end of the experiments, after removal of the electrode array, the maximum force for whole nerve stimulation with a hook electrode proximal to the incision was $10-40 \%$ lower than for stimulation distal to the incision, pointing at partial blocking of neural conduction. In one case, after experiment $\mathrm{d}$, these final forces were found to be approximately $0 \mathrm{mN}$ and $460 \mathrm{mN}$, respectively. Thus in this particular case, conduction of action potentials toward the muscle was completely blocked at the site of insertion. Note, however, that this did not mean that nerve excitation, by the multi-electrode, had disappeared.

The influence of electrode impalement on action potential propagation in peripheral nerves has been studied by Calancie and Stein [3] and Rice et al. [16] (among others). Their results suggest that a (temporary) conduction block occurs in a significant number of myelinated fibers near the site of an endoneural $200 \mu \mathrm{m}$ tungsten wire-microelectrode with a sharpened tip.

The most likely causes of conduction block are either direct pressure or structural damage to neural tissue [16]. The former is believed to be a temporary and reversible phenomenon caused by the pressure of the electrode in the nerve (and not necessarily associated with structural injury to nerve fibers), whereas the latter would be an irreversible effect. As pressure is (probably) absent after removal of the array, the apparent conduction block in our experiments must be mainly ascribed to (acute) neural injury.

\section{Considerations on Theoretical Selectivity}

Efficiency $E$ is a measure that can be easily calculated. The selectivity measure $S$, on the other hand, can not be determined for the experiments presented here. This is due to the fact the experimental procedure does not allow direct identification of single motor units. Therefore, $S$ must be considered a theoretical measure here.

An a priori estimation of the number of (separate) motor units exclusively activated by an endoneural electrode array can be obtained from consideration of the average excitation volume per electrode in combination with anatomical data [11], [17].

The EDL muscle of the rat is innervated by 69 motor fibers in the common peroneal nerve, having an average internodal distance $\lambda$ of $1 \mathrm{~mm}$ [6], [8], [15]. Assuming the fascicle to be a cylinder with a radius $r_{f}$ of $250 \mu \mathrm{m}$, and the nodes of Ranvier to be distributed uniformly inside the fascicle, the $\alpha$-nodal density is $3.5 \times 10^{2} \mathrm{~mm}^{-3}$. Due to the anisotropy of the conductivity, the monopolar electrodes generate ellipsoidal activation regions. Assuming a ratio of longitudinal and radial conductivities $\sigma_{z} / \sigma_{\rho}$ of 5 [8], [11], [18], the excitation volumes are easily calculated.

In the $6 \times 4$ electrode array the electrodes are separated from each other by $120 \mu \mathrm{m}$. The number of nodes of Ranvier exclusively "seen" by each electrode depends on the size of 


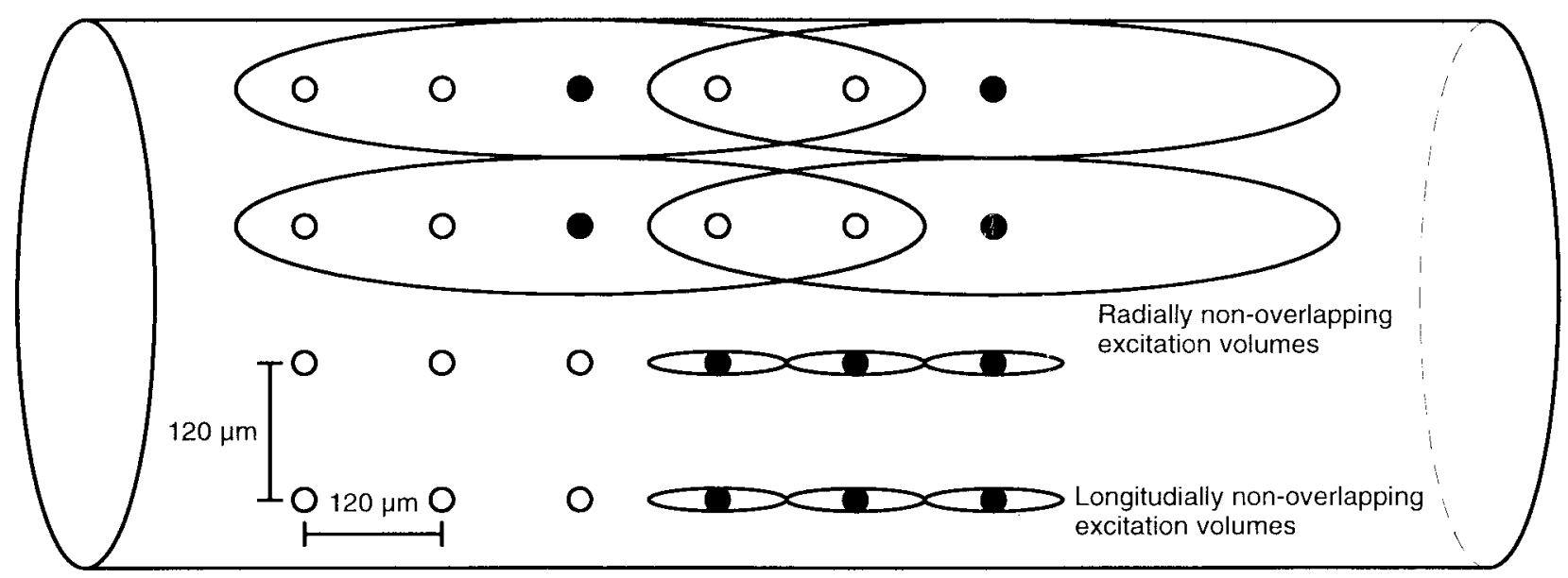

Fig. 6. Schematic representation of excitation volumes for monopolar stimulation in a homogeneous, anisotropic conductive cylinder with a radius rf of $250 \mu \mathrm{m}$ and $\sigma z / \sigma \rho=5$. A $6 \times 4$ electrode array was assumed to be inserted into the fascicle. The electrodes (indicated by the small circles) are separated from each other by $120 \mu \mathrm{m}$. Active electrodes are shown as black circles at the center of an ellipsoidal excitation volume. For longitudinally nonoverlapping volumes, the long axis is limited to $120 \mu \mathrm{m}$ (cf., the two bottom rows of electrodes). For radially nonoverlapping volumes, the short axis is limited to $120 \mu \mathrm{m}$ (cf. the two top rows of electrodes).

the excitation volumes and the amount of overlap between them (Fig. 6). For longitudinally nonoverlapping excitation volumes, the long axis of the ellipsoid is limited to $120 \mu \mathrm{m}$, and the number of nodes of Ranvier activated selectively by an electrode is $1.3 \times 10^{-2}$. The total number of nerve fibers contacted selectively with the 24 electrodes in the array thus is 0.3 . This indicates that overlapping excitation volumes are inevitable if a substantial fraction of the nerve fibers is to be activated.

For radially nonoverlapping excitation volumes (implying the short axis of the ellipsoid to be limited to $120 \mu \mathrm{m}), 1.6$ nodes of Ranvier can be activated by an electrode. The total number activated by the 24 electrodes in the array is thus 38.4. Due to the considerable overlap in longitudinal direction, however, a substantial fraction of this number represents nodes that can be activated by multiple electrodes. Obviously, larger overlap implies lower selectivity.

These examples show that a trade-off has to be made between selectivity of stimulation and total number of nerve fibers that can be activated. For a more realistic estimation of selectivity, the long axis of the excitation volume might be taken three times the interelectrode spacing. This yields 0.34 activated nodes of Ranvier for an electrode. The total number of nerve fibers then contacted with the electrode array is thus 8.2 .

The above considerations clearly indicate that optimal selectivity requires the use of redundant three-dimensional electrode configurations, spanning at least one internodal distance in longitudinal direction.

\section{E. A Closer Look at Efficiency}

Since the currents associated with the threshold forces in Table II are low (typically smaller than $8 \mu \mathrm{A}$ ), overlap of the excitation regions in radial direction will be limited (cf. Fig. 6). This can also be deduced from the excitation regions calculated by Meier for monopolar stimulation with cathodic current pulses [11]. Therefore, threshold forces in one bin
TABLE III

EFFICIENCY $E$ AND INCREASED EFFICIENCY $E^{\prime}$ FOR THE EIGHT WMEA EXPERIMENTS LISTED IN TABLE I

\begin{tabular}{cccc}
\hline Experiment & E & E & No. of electrodes \\
\hline a & 0.80 & 1 & 5 \\
b & 0.40 & 0.80 & 5 \\
$c$ & 0.60 & 0.80 & 5 \\
d & 0.42 & 0.79 & 24 \\
c & 0.50 & 0.88 & 8 \\
f & 0.47 & 0.71 & 17 \\
\hline Average & 0.48 & 0.83 & 14 \\
\hline h & 0.46 & 0.81 & \\
\hline
\end{tabular}

that correspond to electrodes located at positions "radially" far apart in the array, are likely to represent different (groups of) motor units. At the same time, if a motor unit (or a small group of motor units) is activated by different electrodes, these are likely to be neighbors in longitudinal direction.

Analysis of the first bin in Table II reveals three longitudinally neighboring electrode pairs (viz. electrodes 4-5, 7-8, and 15-16). The third bin contains one such pair (viz. electrodes 9-10). This suggests that these bins may actually contain four (7-3) and two (3-1) (small groups of) motor units, respectively, thereby increasing the efficiency $E$ for this 
particular experiment from the previously calculated 11/24 = 0.46 to

$$
E^{\prime}=20 / 24=0.83
$$

The same way, the efficiencies for the other arrays can be determined (Table III). It can be seen that there are two extremes, viz. a high efficiency for experiment a and a low efficiency for experiment $h$. The average efficiencies can be determined to be 0.48 and 0.81 , respectively.

The efficiency of a multi-electrode should not be taken as the only absolute measure for successful array performance. It should always be considered together with the number of electrodes in the array, the statistical distribution of the position of nodes of Ranvier in the fascicle, and the specific use of the array for muscle control. For example, the fivechannel electrode arrays in Table III score about as well as the 24-channel arrays and this should not be misinterpreted. The total number of distinctly addressed threshold forces is very different in both cases! The efficiency being about equal in both groups simply means that the interelectrode spacing is equal. The number of five or 24 electrodes still undersamples the distribution of fibers in the fascicle: the arrays are both far from being redundant.

\section{F. Clinical Application Aspects}

In chronic applications of endoneural stimulation several problems might be encountered. First, stability of electrode positions is demanded to ensure predictable responses to stimulation. This is especially vital when finely graded movements are to be generated. Fixation of multi-electrode arrays is expected to be difficult in peripheral nerves, because of their liability to displacement during muscle contractions. This problem can be dealt with by inserting the electrode arrays more centrally in the nervous system, e.g., in the ventral roots or the spinal cord. The latter option offers the additional advantage of electrode fixation to the vertebrae.

Another problem to be dealt with is the insertion of electrode arrays. Successful insertion should result in the electrodes being positioned between the nerve fibers. Actual insertion of multi-electrodes into peripheral nerves, penetrating the tough epineural and perineural tissue, is not straightforward. Impact insertion was found to be not feasible, since array impulse (or speed) could not be increased to the required level without damaging either array or nerve. This must be ascribed to the bluntness of the array electrodes and the high viscoelasticity of neural tissue. We also have investigated an alternative insertion method based on radio-frequency cutting techniques [20]. However, in the experiments presented here, we used an ad hoc method to facilitate insertion of electrode arrays, viz. creation of an incision in the epineurium and perineurium of the nerve. Approximately 17\% of these insertions were unsuccessful.

In conclusion it can be stated that under carefully monitored experimental conditions selective neuromuscular activation by means of endoneural electrode arrays is feasible. However, chronic experiments have to be done to elucidate the effect of muscle contractions and limb movement on the mechanical and electrical stability of the electrode-nerve contact. Also, long-term effects of insertion methods, such as wound healing and electrode encapsulation, have to be assessed.

\section{ACKNOWLEDGMENT}

The authors would like to acknowledge the help of A. J. Verloop (for manufacturing the wire-microelectrode arrays), and R. Busschers and Dr. W. Wallinga-de Jonge (for preparation of the animals and/or assistance during the experiments) is gratefully acknowledged.

\section{REFERENCES}

[1] R. Baratta, M. Ichie, S. K. Hwang, and M. Solomonow, "Orderly stimulation of skeletal muscle motor units with tripolar nerve cuff electrode," IEEE Trans. Biomed. Eng., vol. 36, pp. 836-843, 1989.

[2] M. D. Binder and L. M. Mendell, Eds., The Segmental Motor System, first ed. Oxford, U.K.: Oxford Univ. Press, 1990.

[3] B. M. Calancie and R. B. Stein, "Microneurography for the recording and selective stimulation of afferents: An assessment," Muscle Nerve, vol. 11, pp. 638-644, 1988.

[4] Z. P. Fang and J. T. Mortimer, "Selective activation of small motor axons by quasitrapezoidal current pulses," IEEE Trans. Biomed. Eng., vol. 38, pp. 168-174, 1991.

[5] _ "A method to effect physiological recruitment order in electrically activated muscle," IEEE Trans. Biomed. Eng., vol. 38, pp. 175-179, 1991.

[6] T. A. Frieswijk and W. L. C. Rutten, "NEVIS: Visualization of factors influencing force recruitment in intraneural stimulation, Part II," in Proc. 16th Ann. Int. Conf. IEEE Eng. Med. Biol. Soc., 1994a, pp. 361-362.

[7] _ , "Three-dimensional neuroelectronic interface for peripheral nerve stimulation and recording: Realization steps and contacting technology," in Proc. 16th Ann. Int. Conf. IEEE Eng. Med. Biol. Soc., pp. 808-809, 1994b.

[8] T. A. Frieswijk, J. P. A. Smit, W. L. C. Rutten, and H. B. K. Boom, "Force-current relationships in intraneural stimulation: Role of extraneural medium and motor fiber clustering," Med. Biol. Eng. Comput., 1998.

[9] W. M. Grill and J. T. Mortimer, "The effect of stimulus pulse duration on selectivity of neural stimulation," IEEE Trans. Biomed. Eng., vol. 43, pp. 161-166, 1996.

[10] A. C. Hoogerwerf and K. D. Wise, "A 3D micro-electrode array for chronic neural recording," IEEE Trans. Biomed. Eng., vol. 41, pp. 1136-1146, 1994.

[11] J. H. Meier, "Selectivity and design of neuro-electronic interfaces," $\mathrm{Ph} . \mathrm{D}$. dissertation, University of Twente, Enschede, The Netherlands, 1992.

[12] J. H. Meier, W. L. C. Rutten, A. E. Zoutman, H. B. K. Boom, and P. Bergveld, "Simulation of multipolar fiber selective neural stimulation using intrafascicular electrodes," IEEE Trans. Biomed. Eng., vol. 39, pp. 122-134, 1992

[13] J. H. Meier, W. L. C. Rutten, and H. B. K. Boom, "Force recruitment during electrical nerve stimulation with multipolar intrafascicular electrodes," Med. Biol. Eng. Comput., vol. 33, pp. 409-417, 1995.

[14] N. Nannini and K. Horch, "Muscle recruitment with intrafascicular electrodes," IEEE Trans. Biomed. Eng., vol. 38, pp. 769-776, 1991.

[15] J. M. Peyronnard, L. F. Charron, J. Lavoie, and J. P. Messier, "Motor, sympathetic and sensory innervation of rat skeletal muscles," Brain Res., vol. 373, pp. 288-302, 1986.

[16] A. S. C. Rice, S. B. McMahon, and P. D. Wall, "The electrophysiological consequences of electrode impalement of peripheral nerves in the rat," Brain Res., vol. 631, pp. 221-226, 1993.

[17] W. L. C. Rutten, H. J. van Wier, and J. H. M. Put, "Sensitivity and selectivity of intraneural stimulation using a silicon electrode array," IEEE Trans. Biomed. Eng., vol. 38, pp. 192-198, 1991.

[18] W. L. C. Rutten, T. A. Frieswijk, J. P. A. Smit, T. H. Rozijn, and J. H. Meier, "3D Neuro-electronic interface devices for neuromuscular control: Design studies and realization steps," Biosensors Bioelectron., vol. 10, pp. 141-153, 1995.

[19] J. P. A. Smit, W. L. C. Rutten, T. A. Frieswijk, and R. Busschers, "The influence of nerve fiber distribution on the recruitment behavior resulting from intraneural stimulation," in Proc. 15th Ann. Int. Conf. IEEE Eng. Med. Biol. Soc., 1993, pp. 1198-1199. 
[20] J. P. A. Smit and W. L. C. Rutten, "A technique for electrically inserting electrode arrays into peripheral nerves," in Proc. 16th Ann. Int. Conf. IEEE Eng. Med. Biol. Soc., 1994, pp. 800-801.

[21] J. D. Sweeney, D. A. Ksienski, and J. T. Mortimer, "A nerve cuff technique for selective excitation of peripheral nerve trunk regions," IEEE Trans. Biomed. Eng., vol. 37, pp. 706-715, 1990.

[22] P. H. Veltink, J. A. van Alsté, and H. B. K. Boom, "Multielectrode intrafascicular and extraneural stimulation," Med. Biol. Eng. Comput., vol. 27, pp. 19-24, 1989.

[23] C. Veraart, W. M. Grill, and J. T. Mortimer, "Selective control of muscle activation with a multipolar nerve cuff electrode," IEEE Trans. Biomed. Eng., vol. 40, pp. 640-653, 1993.

[24] W. Wallinga-De Jonge, H. B. K. Boom, K. L. Boon, P. A. M. Griep, and G. C. Lammeree, "Force development of fast and slow skeletal muscle at different muscle lengths," Am. J. Physiol., vol. 239, pp. C98-C104, 1980.

[25] K. Yoshida and K. Horch, "Selective stimulation of peripheral nerve fibers using dual intrafascicular electrodes," IEEE Trans. Biomed. Eng., vol. 40, pp. 492-494, 1993.

[26] _ _ "Closed-loop control of ankle position using muscle afferent feedback with functional neuromuscular stimulation," IEEE Trans. Biomed. Eng., vol. 43, pp. 167-176, 1996.



Jos P. A. Smit was born in Aalten, The Netherlands, in 1965. He received the M.Sc. degree in electrical engineering and the Ph.D. degree in biomedical engineering both from the University of Twente, Enschede, The Netherlands, in 1989 and 1996, respectively.

Following a Postdoctoral position at the University of Twente, he joined the Research Department of Vitatron Medical B. V., Dieren, The Netherlands, in 1998. As a Clinical Software Engineer, he is involved in the development of new therapeutic and diagnostic tools in cardiac pacing and in conducting clinical feasibility studies. His research interests include (selective) electrical stimulation and cardiac pacing.



Wim L. C. Rutten was born in 1950 . He was trained as experimental physicist and received the Ph.D. degree from Leiden University, The Netherlands, in 1979.

He studied the auditory system at the ENT Department, Leiden University Hospital, The Netherlands, and since 1985, he has been with BMTI/Faculty of Electrical Engineering, University of Twente, Enschede, The Netherlands, as an Assistant and Associate Professor of Biomedical Control Systems. His present research interests are neural engineering and cellular engineering (neuro-electronic interfaces, cultured probes) signal processing, and bioelectricity.

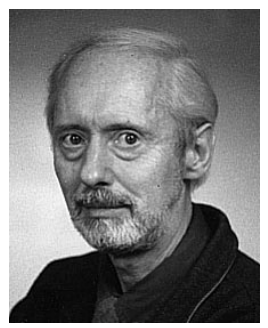

Herman B. K. Boom received the Ph.D. degree at the University of Utrecht, The Netherlands, in 1971, where he was trained as a Medical Physicist.

He joined the Department of Medical Physics and Medical Physiology, where he was engaged in research in the field of cardiac mechanics and taught physiology and biophysics. Since 1976, he has been a Professor of Medical Electronics in the Department of Electrical Engineering, University of Twente, Enschede, The Netherlands. His research bioelectricity, and rehabilitation technology. 